\title{
A Study of Internet Development and Enterprise Financing in China
}

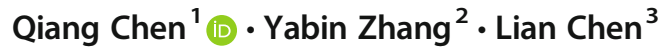

Accepted: 9 April 2021/ Published online: 7 May 2021

(C) The Author(s) 2021

\begin{abstract}
The development of the Internet has changed many traditional commercial models of enterprises. Therefore, the term Internet development is increasingly drawing attention from scholars. However, we are not certain whether the development of the Internet improves the problem of difficult financing of enterprises and enhances enterprise financing efficiency. This paper analyzes whether Internet development can promote enterprise financing efficiency. First, the Data Envelopment Analysis- Slack Based Measure (DEA-SBM) model is applied to measure the enterprise financing efficiency values of 31 provinces in China. The measurement results indicate that the enterprise financing efficiency in the eastern regions is higher than that of central and western regions. Then, this paper further tests if Internet development can contribute to enterprise financing efficiency based on an econometric model. The analysis results indicate that Internet development has an obvious promoting effect on enterprise financing efficiency. Later, spatial econometric models are adopted in this paper to further analyze if Internet development has a spatial overflow effect on enterprise financing efficiency. The results indicate that Internet development indeed has a spatial overflow effect on enterprise financing efficiency.
\end{abstract}

Keywords Internet development - Enterprise financing $\cdot \mathrm{DEA}-\mathrm{SBM} \cdot$ Spatial econometrics

\section{Introduction}

Since its entry into China, the Internet has already developed from a "fresh product" in the very beginning to a "necessity" for people in their work and lives. Meanwhile, the

Qiang Chen

paperway@sina.cn

1 Guangdong Academy of Agricultural Sciences, Guangzhou 510640, China

2 Hunan University, Changsha 410082, China

3 Southwest University of Political Science and Law, Chongqing 401120, China 
Internet's functions have also developed from network communication and social networking at the very beginning to commercial networking, resource sharing, service cloud-based service, and other relevant models. Currently, the Internet economy generated by Internet technology has gradually become a new economic form after the agricultural economy and industrial economy. With the development of the Internet, Internet finance (a financing model based on e-commerce) has come into being. Hence, scholars have begun to pay close attention to the relationship between the Internet and economic growth.

Koutroumpis (2009) (Koutroumpis 2009) found that broadband infrastructure and economic growth have a significant positive relationship. Pradhan (2016) (Pradhan et al. 2016) found that Internet penetration rates and economic growth have a causal relationship. Bailey (1997) (Bailey and Mcknight 1997) thought that the Internet economy was a growing area of concern for members of the technical, business, academic, and user communities. Li (2014) (Haijian et al. 2014) carried out a transitional summarization of the Internet economy's development in terms of Internet spirits, concepts and economics and concluded that great changes would occur in the technical, structural and performance levels of transactions. Tian (2014) (Guangning and Wang 2014) believed that the Internet economy changed the most essential transaction model of traditional economy, and realized the completion of information flows, capital flows and transaction logistics through the Internet. Cheng (2013) (Cheng 2013) thought that the Internet economy was essentially an innovative economy. Therefore, the reason why the Internet can change many traditional commercial models mainly lies in its unique features of high speed information spreading, an extensive information spreading scope and an information exchange platform. In a market economy, enterprises are the subject of the market economy, while capital is the main driving force for enterprise development. To this end, the issue of enterprise financing is the key to enterprise development (Erel, 2015; Yuan, 2016; Lennox, 2017) (Erel et al. 2015; Yuan et al. 2016; Lennox et al. 2017). Modigliani and Miller (1958) (Modigliani and Miller 1958) proposed the Modigliani \& Miller (MM) theory, which spurred the beginning of modern corporate finance theory. This theory states that in a perfect market, the enterprise capital structure and corporate market value have no effect. Modigliani and Miller (1963) (Modigliani and Miller 1963) found that more debt led to more obvious effects of leverage and a larger company value. Information asymmetry has always been an important factor that influences enterprises' financing efficiency. Many scholars have used the perspective of information asymmetry to research corporate financing problems. Rajan (1992) (Rajan 1992) found that the reduction of enterprise financing costs mainly depends on the solution of the asymmetric information problem. Kaplan(1997) (Kaplan and Zingales 1997) believed that information asymmetry between enterprises and the external investors was one of the important causes that led to enterprise financing constraints. Cheng (2014) (Cheng et al. 2014) found that high quality information disclosure and analyst predictions can reduce the information asymmetry between internal management and external capital providers. Enterprises are the mainstay of economic development, and financing is the main issue for enterprise development.

Although the development of the Internet changes many traditional commercial models and improves benefits of enterprises, how the development of the Internet affects enterprise financing still needs further research. There is a lack of literature 
examining the field of Internet and enterprise financing. Thus, this article will study the influence of Internet development on enterprise financing efficiency. In recent years, spatial analysis has become an important approach for the analysis of regional differences (Grange, 2012; Griffith, 2015) (Grange et al. 2012; Griffith and Chun 2015). This paper will use spatial analysis methods to analyze the development of the Internet's spatial spillover effects on enterprise financing efficiency. This paper will consider the transaction efficiency as financing efficiency. In the calculation of financing efficiency, a large number of studies have used Data Envelopment Analysis (DEA) approaches (Charnes, 1978) (Charnes et al. 1978) to calculate the financing efficiency (Zhao, 2011; Lin, 2013; Wang, 2017) (Shouguo et al. 2011; Yu et al. 2013; Wang et al. 2017). Therefore, this paper will also use DEA approaches to calculate the financing efficiency.

The structure of this paper is as follows. Section 1 is the introduction. Section 2 introduces the theoretical analysis and assumptions. Section 3 introduces the research methods and materials, Section 4 describes the results, and Section 5 provides the conclusions and suggestions.

\section{Theoretical Analysis and Assumptions}

Although there is no uniform definition of financing efficiency, financing efficiency mainly includes the two aspects of transaction efficiency and capital allocation efficiency (Han, 2016; Zhang, 2017) (Dobrovolsky 1958; Myers and Majluf 1983). Transaction efficiency refers to the ability of enterprises to obtain capital at the lowest costs. Allocative efficiency refers to the fact that enterprises will maximize the utilization of raised funds. There are usually two enterprise financing methods, internal financing and external financing (Dobrovolsky, 1958) (Xuwen 2016). Internal financing refers to financing that is directly acquired by enterprises. External financing refers to enterprise financing by means of issuing of bonds, sale of equity, and other such methods. Myer (1983) (Xinmin et al. 2017) proposed the Pecking Order Theory, in which enterprise financing generally follows this order: retained profits, debt financing and equity financing. Retained profits are internal financing. Debt financing and equity financing are external financing. In particular, debt financing is the main and commonly used financing method. Therefore, the external financing is an important enterprise financing method, especially listed enterprise ( $\mathrm{Li}, 2003$ ) (Yichao 2003). In the process of external financing, enterprises often face many problems, such as information asymmetry and high transaction costs. In this paper, it is concluded that Internet development influences the efficiency of enterprise financing mainly through information symmetry and transaction costs.

Before the advent of the Internet, both large enterprises and SMEs would encounter information asymmetry due to restrictions such as enterprise scale and industrial scope. Information asymmetry can limit the enterprise financing channels and increase the transaction costs of enterprise financing (Li, 2015) (Zhiqiang 2015). Furthermore, information asymmetry can also limit the information collection channels regarding how the capital suppliers understand the loan enterprises in order to increase the transaction costs of capital suppliers. Moreover, since many capital suppliers cannot collect complete information regarding every financing enterprise, many financing enterprises will be shut out (Wang, 2009) (Wang 2009). 
After the advent of the Internet, since the Internet features the integration of information resources, large information spreading and an extensive information spreading scope, its development has effectively accelerated the conversion of information asymmetry to information symmetry in the financing process, which could improve the information transparency of financing enterprises and capital suppliers in the financing process in order to avoid the occurrence of problems such as the "Lemon Market" (Akerlof, 1970) (Akerlof 1970). Under the condition that information asymmetry is changed, enterprises are able to acquire more financing information, and the enterprises are able to change from the original bounded rational man to the perfectly rational man during the process of financing. As a result, enterprises can make more rational choices. In addition, the financial development of the Internet has simplified the operation flow and management architecture of traditional financial service. Meanwhile, unnecessary intermediate processes are reduced in the financial industrial chain. The financing efficiency of enterprises and financial institutions are improved, and the transaction costs of enterprise financing are lowered (Liu, 2019) (Manfeng and Long 2019). Therefore, the Internet provides enterprises with more extensive financing channels, it solves the issue of information asymmetry during the process of enterprise financing, and it also breaks the monopolistic position of traditional channels. Moreover, the Internet has reduced many intermediate financial processes. Meanwhile, the requirements of Internet financing are relatively flexible, which can further lower the threshold of enterprise financing, reduce the transaction costs of enterprise financing and improve the financing restraints of enterprises. If viewed from a macro perspective, the Internet could gather statistics and manage the financing demands with proper timing in order to better match market liquidity with financing demands and avoid the occurrence of credit rationing (Stiglitz \& Weiss, 1981) (Stiglitz and Weiss 1981).

The Internet has the characteristics of fast transmission speed and wide range. Therefore, the Internet has a strong spatial effect. Moreover, due to inter-regional financial trade, the financing efficiency of local enterprises will also be affected by other regions. As a medium of enterprise financing, the Internet will promote the penetration and diffusion of local financing efficiency to enterprise financing efficiency in other regions. In addition, due to the different levels of Internet development in different regions (Zheng, 2016) (Du Zheng Siqi and Weizeng 2016), the spatial diffusion effect of the Internet on enterprise financing will be different.

Hence, the following theoretic assumptions are proposed in this paper. The development of the Internet has a promoting effect on enterprise financing efficiency. In other words, with the development of Internet, enterprise financing efficiency will be accordingly improved. Meanwhile, in consideration of the imbalanced development of the Internet and the information overflow effect of the Internet itself, this paper concludes that Internet development has a spatial overflow effect on enterprise financing efficiency.

\section{Materials and Methods}

\subsection{Calculation of Enterprise Financing Efficiency Value}

In this paper, Data Envelopment Analysis is applied to measure enterprise financing's efficiency. Meanwhile, since traditional models such as the Charnes Cooper Rhodes 
(CCR) model and the Banker Charnes Cooper (BCC) model are relatively frequently used in DEA and they fail to take unexpected output into account, SBM based on the non-radial and non-angular model proposed by Tone (2001) (Tone 2001) is selected and used in this paper. SBM considers the unexpected output index. Since this paper selects the financing costs as the unexpected output, this model is more suitable. DEAMAX, a DEA software, would be used to measure the enterprise financing efficiency. The DEA-SBM model applied in this paper is shown as follows:

$$
\begin{gathered}
\min \rho=\frac{\frac{1}{m} \sum_{i=1}^{m} s_{i}^{-} / x_{i k}}{1+\frac{1}{q} \sum_{r=1}^{q} s_{r}^{+} / y_{r k}} \\
\text { s.t. } X \lambda+s^{-}=x_{k} \\
Y \lambda+s^{+}=y_{k} \\
\lambda, s^{-}, s^{+} \geq 0
\end{gathered}
$$

Here, min $\rho$ represents the enterprise financing efficiency. y represents the output vectors of the Decision Making Unit (DMU). y represents the input vectors of the DMU. $\mathrm{s}^{+}$and $\mathrm{s}^{-}$represent the slack variables. $\lambda$ represents the weights of the DMU.

\subsection{Econometric Analysis}

\subsubsection{SLS Model}

In consideration of the endogenous issues, the two-stage least square (2SLS) (Wooldridge, 2014) (Wooldridge 2014) was used in this paper to test the influence of Internet development on enterprise financing efficiency. Stata, a statistical software, would be used as the analysis of regression. The econometric model used in this paper is introduced as follows:

$$
\mathrm{Y}_{\mathrm{i}, \mathrm{t}}=\alpha+\beta * \mathrm{NET}_{\mathrm{i}, \mathrm{t}}+\sum_{i=2}^{n} \beta_{i} Z_{i t}^{n}+\varepsilon_{\mathrm{i}, \mathrm{t}}
$$

Here, Y represents the enterprise financing efficiency. $\alpha$ represents the intercept. $\beta$ represents the slope. NET represents the Internet's development. $Z$ represents the control variables, which includes the Government Management, Property Right Structure, Openness Level, Financial Level and Infrastructure Level. $\varepsilon$ represents the disturbance.

\subsubsection{Spatial Econometric Analysis}

The spatial econometric model is further selected and used in this paper to analyze if Internet development has a spatial overflow effect on enterprise financing efficiency. The Spatial Autoregressive model (SAR) (Elhorst, 2014) (Elhorst 2014) is selected and used accordingly to model the test result. The research would select Matlab, a statistical software, as the regression analysis tool. The spatial error model is shown as follows: 


$$
\mathrm{Y}_{\mathrm{i}}=\lambda w Y_{i}+\beta \mathrm{X}_{\mathrm{i}}+\varepsilon_{i}
$$

Here, $\mathrm{Y}$ represents the enterprise financing efficiency. $\mathrm{X}$ represents the explanatory variables. $\lambda$ represents the regression coefficient of autocorrelation. $w$ represents the weight matrix. $\beta$ represents the estimated parameters. $\varepsilon$ represents the disturbance.

The spatial econometric model is further selected and used in this paper to analyze if Internet development has a spatial overflow effect on enterprise financing efficiency. The Spatial Error model (SEM) (Elhorst 2014) is selected and used accordingly to model the test result. The research would select Matlab, a statistical software, as the regression analysis tool. The spatial error model is shown as follows:

$$
\mathrm{Y}_{\mathrm{i}}=\beta_{k} x_{k}+\mu_{i}+u_{i}, u_{i}=\lambda \sum_{j=1}^{N} w_{i j} u_{j}+\varepsilon_{i}
$$

Here, Y represents the enterprise financing efficiency. $\lambda$ represents the parameters of estimation. $\mathrm{X}$ represents the independent variable and other control variables. $\mathrm{W}$ represents the weight matrix. $\mu$ represents the specific effect of space. $\varepsilon$ represents the disturbance.

\subsection{Data}

\subsubsection{DEA Data}

In the aspect of data selection, we refer to the previous studies (Liu, 2004; Zhang, 2014; Li, 2014) (Lichang et al. 2004; Quan 2014; Fang and Wang 2014). The paper takes the shareholders' equity and the Tobin's Q as the input index, and the total debt and the sum of administrative expenses and financing expenses are used as the output index. Meanwhile, the sum of administrative expenses and financing expenses is the bad output. This paper calculates the enterprise financing efficiency. The data of this paper are sourced from 2867 listed companies from the Shanghai stock exchange and the Shenzhen stock exchange from 2006 to 2013 in China. According to the registered address, the paper incorporates these companies into their region. The data on financing efficiency mainly come from the China Stock Market \& Accounting Research Database (CSMAR) and the Wind database. Since the data in some regions are insufficient, the data are sourced from 31 provinces from mainland China (excluding Taiwan, Hong Kong and Macao).

\subsubsection{Econometric Data}

The data cover 31 total regions, including the eastern, central and western regions in China. In the econometric analysis, the core variables of this paper are the enterprise financing efficiency and Internet development. The control variables include the variable regarding the structure of property rights, the openness level, the credit level and the infrastructure level. The variable of the enterprise financing efficiency has been calculated. The variable of Internet development includes the sum of Internet 
proportion, the per capita broadband use and the employed proportion of information transmission, computer services and software. The instrumental variable is the Internet development lagged one period. The variable for the government management is the ratio of the sum of fiscal income and fiscal expenditure and Gross Domestic Product (GDP). The variable regarding the structure of property rights uses the ratio of the stateowned companies and large companies. The variable for the openness level is the ratio of foreign investment and GDP. The financial level uses the number of financial employees. The variable for the infrastructure level uses the length of traffic operation. Based on the theory of transportation costs (Samuelson, 1952) (Samuelson 1952), distance and transportation costs were considered to be important factors for regional research. However, just considering geographical distance is not enough. At the economic level, also need to consider economic distance. Based on these considerations, this article plans to combine the two important factors of geographic and economic development level. GDP is an authoritative indicator of economic development. GDP is an authoritative indicator of economic development. GDP is an authoritative indicator of economic development. Hence, this paper uses the ratio of the distance between regional centers and the regional GDP to be the spatial weight matrix.

The data are sourced from the statistical yearbook of China, the transportation yearbook of China, the statistical yearbook of investment in fixed assets of China and the Wind Database. Since the data in some regions are insufficient, the data are sourced from 31 provinces from mainland China (excluding Taiwan, Hong Kong and Macao).

\section{Results}

\subsection{Enterprise Financing Efficiency Value}

The calculation results of enterprise financing efficiency show that many regions have seen significant improvements. Particularly, the financing efficiency of enterprises in the eastern region is significantly higher than that in other regions. Figures 1, 2, 3 illustrate the enterprise financing efficiency values and the average values from 2006 to 2013 calculated in this paper. According to the distribution of efficiency values, they are classified into three levels in this paper. Low is indicated by yellow, medium is indicated by blue, and high is indicated by orange. The distribution intervals of efficiency values include $(0,0.13],(0.13,0.17)$ and $(0.17,1]$. Figure 1 shows that the enterprise efficiency values of other provinces in eastern regions except for Shandong Province and Hebei Province are at the medium level and above from 2006 to 2013. For central regions, the enterprise efficiency values of most regions are not significantly improved, and they are still at relatively low levels. Financing efficiency is obviously improved in Neimenggu, Jilin Province and Hubei Province. For western regions, the financing efficiency of most regions is obviously improved, especially in Xinjiang and Qinghai Provinces where a relatively high financing level is presented. The enterprise financing efficiency of eastern regions is at the medium level and above. If viewed from the distribution of average values over the years, the overall enterprise financing efficiency in eastern regions is obviously higher than that in central and western regions. To be specific, enterprise financing efficiency in southeast coastal regions is 
higher than that in other eastern regions, which is directly related to the fact that these regions are located in China's most competitive Yangtze River Delta and Pearl River Delta areas. The enterprise financing efficiency in central and western regions are obviously lower than that in eastern regions as a whole, which is due to the economic development level of central and western regions lagging behind that of eastern regions, and consequently, eastern regions have more resources to invest in infrastructure such as the Internet. Remarkably, the enterprise financing efficiency in western regions is higher than that in central regions. Although the economic development level of central regions is higher than that of western regions, the central regions still lag behind eastern regions as a whole in this aspect. Since the economic development models and industrial structures of the central regions and eastern regions are more similar compared with those of western regions and since eastern regions face the problems of industrial structural adjustments and industrial transfer, more capital should be invested in infrastructure during the economic development of central regions, especially when undertaking industrial transfer from eastern regions. The investment scale of infrastructure is large, and the cost recovery period is relatively long. Therefore, it directly influences the capital turnover of enterprises in central regions and enterprise financing efficiency. The western regions have no geographical advantage for economic development compared to the central regions. Therefore, western regions still mainly focus on the development of more environmentally friendly industries, such as energy and agriculture. Meanwhile, western regions offer certain preferential policies in terms of taxation and more compared with central regions. For example, with respect to the reduction of peasants' tax bearing and the decreased non-tax burden of enterprises released in market-oriented progress data per region (Fan, 2011) (Gang et al. 2011), western regions were superior to central regions.

Figure 4 shows that the enterprise financing efficiency of Eastern regions is obviously higher than that of central regions and western regions. The overall enterprise financing efficiency has grown at a high speed since 2006. It presented a stable growth trend from 2007 to 2013. The enterprise financing efficiency values of the central regions and western regions are relatively close. In addition, the financing efficiency of both the central regions and western regions are both lower than the overall financing efficiency. During the period from 2006 to 2013, the enterprise financing efficiency values of central regions and western regions were not markedly improved. Since the efficiency values of central regions and western regions are close, the central and western regions are combined into one sample for empirical analysis in this paper.

\subsection{Econometric Analysis of Influence of Internet Development on Enterprise Financing Efficiency}

In this paper, the relevant data from the whole country (excluding Hong Kong, Macao and Taiwan), the eastern regions and the central and western regions are selected. An econometric model is applied to analyze the correlation of Internet development with enterprise financing and test the assumptions mentioned above (i.e., whether Internet development has a promotional effect on enterprise financing efficiency). If such a promotional effect exists, this paper will further test if spatial overflow effects exist. If correlation exists, the spatial econometric model will be applied to analyze the spatial overflow effect of Internet development on enterprise financing efficiency and test 


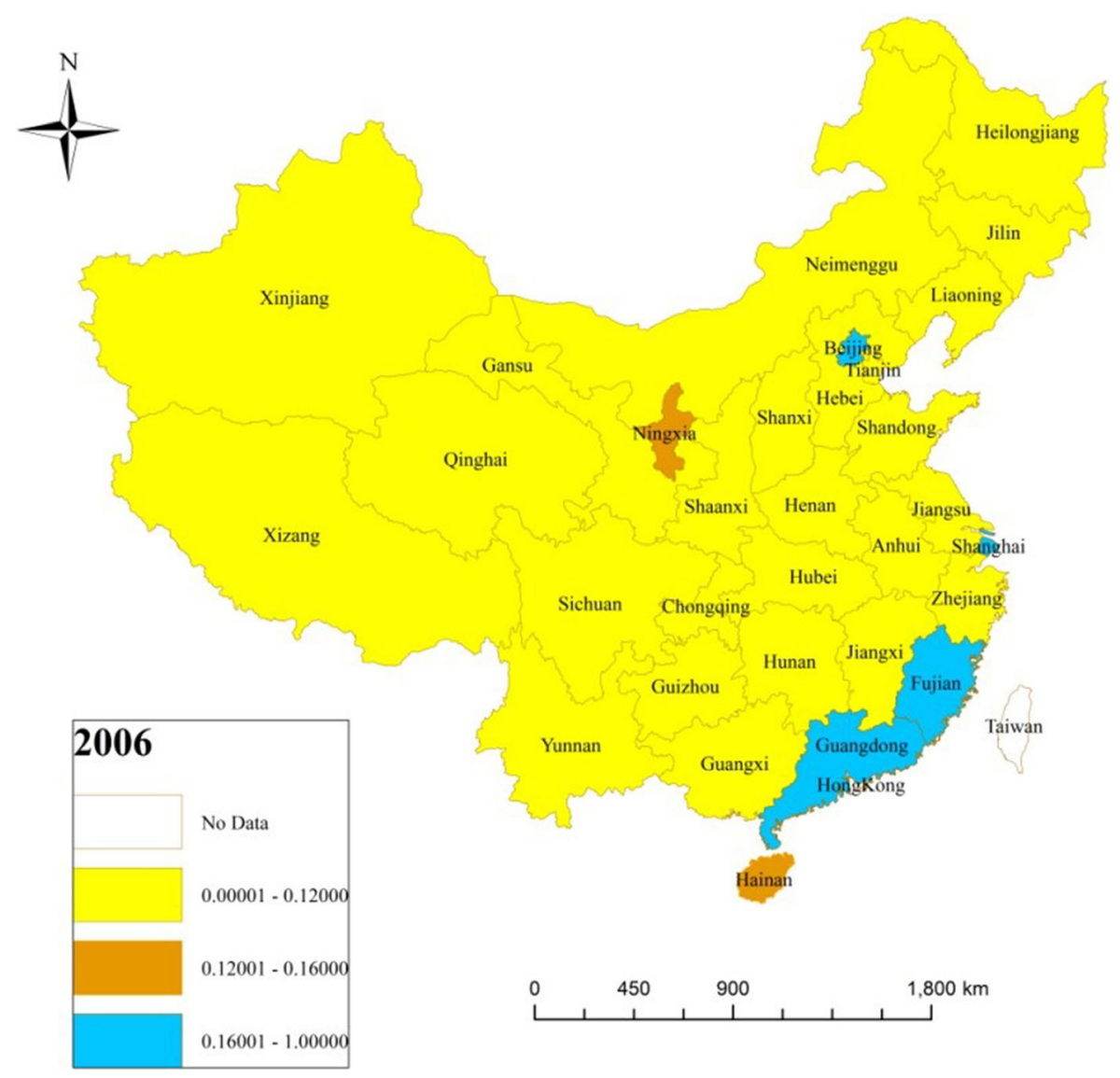

Fig. 1 Enterprise Financing Efficiency of each province (2006)

another assumption of this paper (the spatial overflow effect of Internet development on enterprise financing efficiency). The following is the statistical description of the variables.

Table 1 shows the statistical description. In consideration of the obvious endogenous issues in Internet development, certain instrumental variables shall be required in this paper to handle these issues. The basic idea of the selection of instrumental variable is to choose an exogenous variable that affects an explained variable (enterprise financing efficiency) through an endogenous variable (Internet development) alone. This paper analyzes the exogenousness of selecting the lagging variables of Internet development as the instrumental variable, and found that the lagging variable can significantly impact on the explained variable only through the current variable, and can not directly significantly impact on the explained variable. Therefore, the Internet development variable lagged one phase is selected in this paper as an instrumental variable suitable for Internet development. Table 2 reports the regression results for the first stage of the 2SLS model to test the effectiveness of the instrumental variable. The results indicate that the lagged variable of Internet development has a significant positive influence on Internet development indexes. Moreover, F-test values of the weak instrumental 


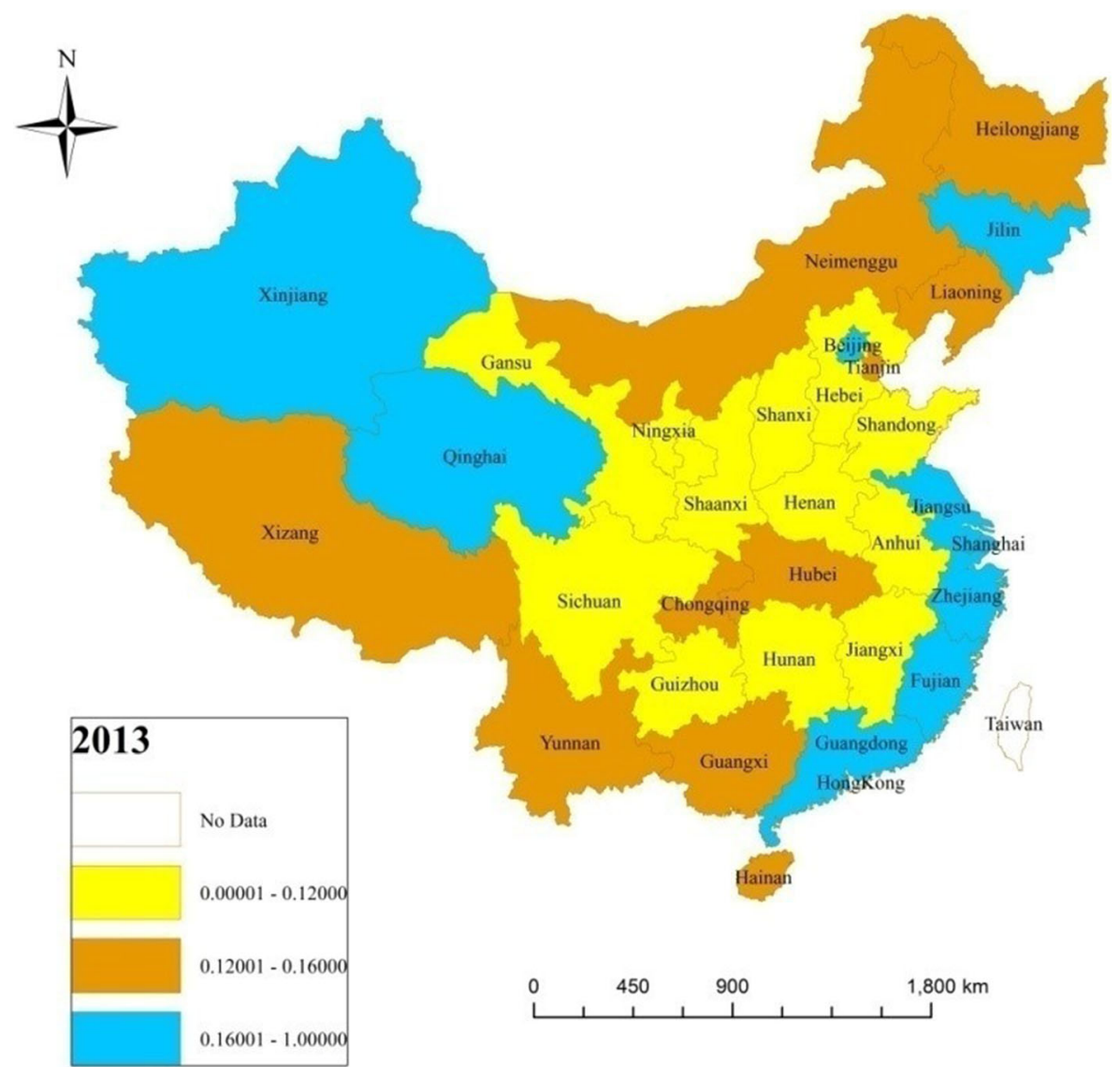

Fig. 2 Enterprise Financing Efficiency of each province (2013)

variables are 3556.3, 1467.48 and 8441.24, respectively. Therefore, the selected instrumental variables have passed the F-test. The abovementioned analysis and results indicate that it is relatively suitable to use the lagged variable of Internet development as the instrumental variable.

Table 3 reports the analysis results of the second stage of the 2SLS model. We can see from the national analysis that Internet development has a significant forward influence on enterprise financing efficiency. This result verifies the assumption of this paper above that Internet development has a promotional effect on enterprise financing efficiency. The analysis results of the eastern and midwest regions also indicate that Internet development has a relatively significant forward influence on enterprise financing efficiency. In addition, the influence of the midwest region is higher than the influence of the eastern region. This finding indicates that, compared with central and western regions, the eastern regions are relatively economically developed with relatively high degrees of marketization. In regions with relatively high levels of marketization, production factors have relatively high liquidity. It is often the case that further disclosures of information are needed to enable the factors to realize highly efficient optimization and configuration of market demands in a timely manner. Thus, 


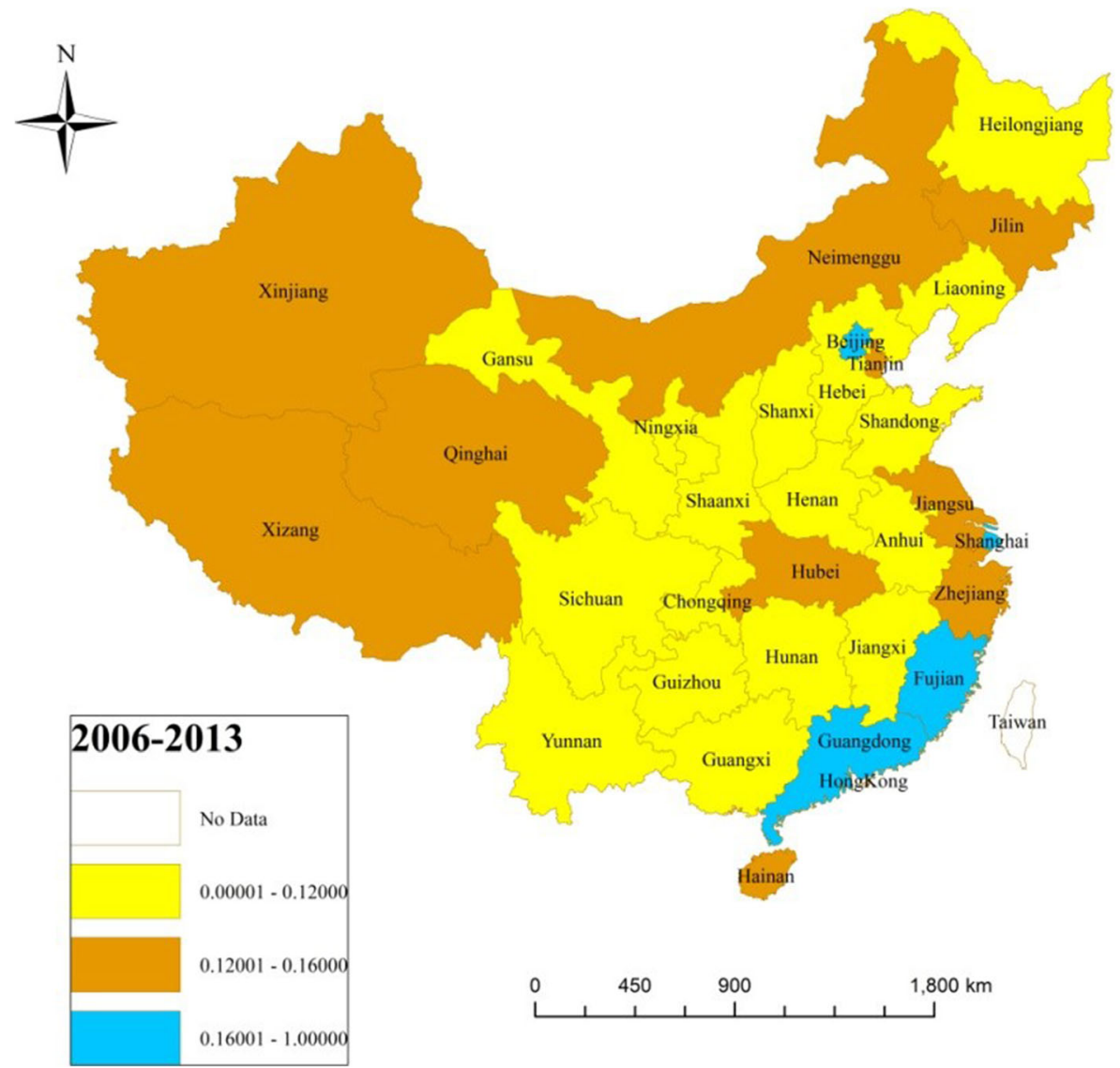

Fig. 3 Enterprise Financing Efficiency of each province (2006-2013)

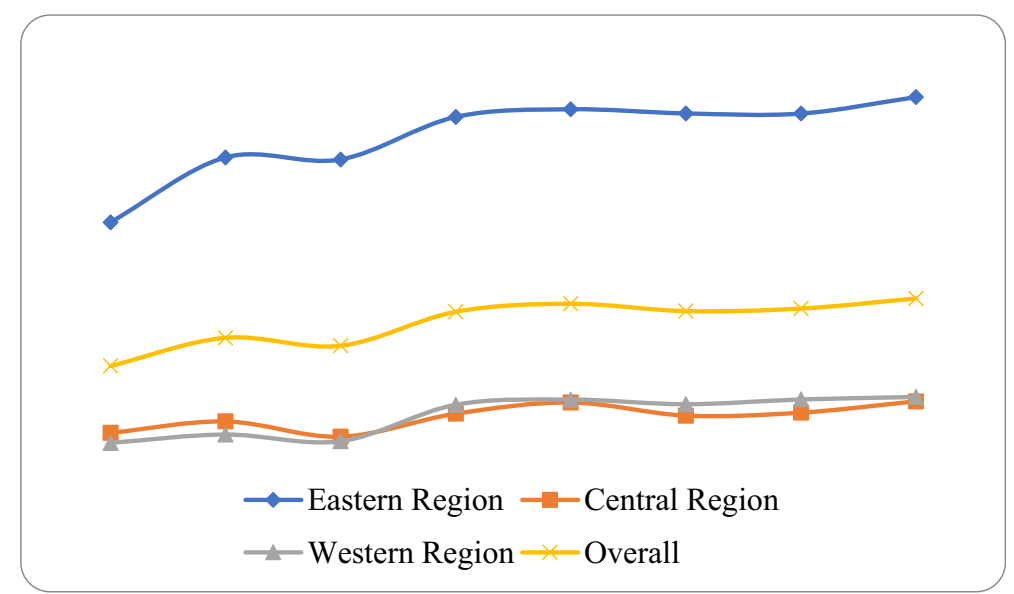

Fig. 4 The enterprise financing efficiency (2006-2013) 
Table 1 Descriptive Statistics

\begin{tabular}{lllllll}
\hline Variable & Symbol & Observation & Mean & Standard Error & Minimum & Maximum \\
\hline Financing Efficiency & EFF & 248 & 0.197 & 0.222 & 0.058 & 1.000 \\
Internet Development & NET & 248 & 0.446 & 0.258 & 0.101 & 0.427 \\
Government Management & CZ & 248 & 0.095 & 0.029 & 0.050 & 0.188 \\
Property Right Structure & GQ & 248 & 0.127 & 0.102 & 0.011 & 0.789 \\
Openness Level & WS & 248 & 0.355 & 0.510 & 0.049 & 4.779 \\
Financial Level & GZ & 248 & 14.710 & 9.835 & 0.740 & 47.590 \\
Infrastructure Level & DL & 248 & 0.110 & 0.034 & 0.057 & 0.248 \\
Internet Development & GJ & 248 & 0.372 & 0.246 & 0.043 & 1.305 \\
(Lag Variable) & & & & & & \\
\hline
\end{tabular}

the influence of Internet development in the midwest region is higher than the influence of Internet development in the eastern region. It is often the case that further disclosures of information are needed to enable the factors to realize highly efficient optimization and configuration of market demands in a timely manner. However, the development of central and western regions lags behind that of eastern regions. Meanwhile, the degree of marketization is also relatively low, and thus, the free liquidity of factors in central and western regions is also not high. The Internet can accelerate the information openness of the upper Midwest and influence the development in central and western regions from many aspects (such as the economic level, the marketization degree and more). Therefore, the effects of the Internet in central and western regions are obviously higher than that in eastern regions. Since the efficiency of enterprise financing and the effect of Internet development on the efficiency of enterprise financing both differ regionally, this paper will use the spatial econometric approaches to test the spatial spillover effect.

Table 4 reports the results of the spatial econometric analysis. The results of the Moran test show that there is a spatial correlation. Based on the LM test result, it is found that it is relatively suitable to select the Spatial Autoregressive Model (SAR) and the Spatial Error Model (SEM). We see from the result of Hausman test that it is relatively suitable to select the fixed effect model at the national level, the random effect model for the Spatial Autoregressive Model and the fixed effect model for the Spatial Error Model, respectively. The regression results from both the SAR model and the

Table 2 The First Stage Result

\begin{tabular}{llll}
\hline & Total & Eastern Region & Midwest Region \\
\hline Internet Development (Lagged Variable) & $1.051^{* * *}$ & $1.019 * * *$ & $1.075 * * *$ \\
Standard Error & 0.018 & 0.027 & 0.012 \\
Observations & 248 & 96 & 152 \\
R-Square & 0.977 & 0.966 & 0.980 \\
F-Test & $3556.300^{* * *}$ & $1467.480^{* * *}$ & $8441.240 * * *$ \\
\hline
\end{tabular}

Note: $* * *, * *$ and $*$ represent significance at the $1 \%, 5 \%$ and $10 \%$ levels, respectively 
SEM model indicate that Internet development has a significant positive influence on enterprise financing efficiency. This proves the assumption of this paper that Internet development has a spatial overflow effect on enterprise financing efficiency. The Internet can effectively improve the local enterprise financing efficiency and promote the enterprise financing efficiency of close regions.

\section{Conclusions and Suggestions}

\subsection{Conclusions}

The research emphasis of this paper is determining whether Internet development can promote enterprise financing efficiency. First, the enterprise financing efficiency values of 31 regions in China are measured. The results indicate that a great gap of enterprise financing efficiency exists between eastern regions and central and western regions. In eastern regions, the enterprise financing efficiency is relatively high, while in central and western regions, it is relatively low. Later, the 2SLS model is further adopted to test the first assumption of this paper that Internet has a promoting effect on improvement of enterprise financing efficiency. The empirical results indicate that the Internet has a significant positive influence on enterprise financing efficiency at the national level. This result confirms the first assumption of this paper. For the regional analysis, the Internet development of eastern regions has an obvious promotional effect on enterprise financing efficiency, while the Internet development of central and western regions does not have an obvious promotional effect on the improvement of enterprise financing efficiency. It is found during further analysis that in central and western regions, the fiscal policies variable has a significant promotional effect on enterprise financing efficiency in central and western regions, and the promotional effect is obviously higher than that of eastern regions. This finding implies that relatively big differences exist between eastern regions and central and western

Table 3 The Second Stage Result

\begin{tabular}{llll}
\hline & Total & Eastern Region & Midwest Region \\
\hline NET & $0.090^{* * *}(0.026)$ & $0.138^{* *}(0.053)$ & $0.064 * *(0.017)$ \\
CZ & $-0.265(0.288)$ & $-0.092(0.867)$ & $-0.032(0.161)$ \\
GQ & $-0.067(0.065)$ & $0.017(0.214)$ & $-0.088^{* * *}(0.032)$ \\
WS & $0.004(0.007)$ & $0.006(0.012)$ & $-0.006(0.033)$ \\
GZ & $0.003^{*}(0.001)$ & $0.001(0.002)$ & $-0.001 * *(0.002)$ \\
DL & $-0.247 *(0.146)$ & $-0.464(0.340)$ & $0.034(0.087)$ \\
Constant & $0.175^{* * *}(0.043)$ & $0.279 * *(0.118)$ & $0.119 * * *(0.018)$ \\
Observations & 248 & 96 & 152 \\
R -square & 0.163 & 0.126 & 0.225 \\
\hline
\end{tabular}

Note: $* * *, * *$ and $*$ represent significance at the $1 \%, 5 \%$ and $10 \%$ levels, respectively. The standard error is in brackets 
Table 4 Spatial Econometric Result

\begin{tabular}{|c|c|c|c|c|}
\hline & SAR (Random) & SEM (Time-Fixed) & SEM (Space-Fixed) & SEM (Space\&Time-Fixed) \\
\hline NET & $0.081 * * *(0.027)$ & $0.843 * * *(0.095)$ & $0.143^{* * * *}(0.029)$ & $0.222 * * *(0.041)$ \\
\hline $\mathbf{C Z}$ & $-0.354(0.271)$ & $0.245(0.474)$ & $-0.267(0.277)$ & $0.178(0.307)$ \\
\hline GQ & $-0.019(0.062)$ & $-0.026(0.125)$ & $0.036(0.071)$ & $0.022(0.080)$ \\
\hline WS & $0.003(0.007)$ & $0.004(0.021)$ & $0.002(0.007)$ & $0.003(0.007)$ \\
\hline GZ & $0.002 * *(0.001)$ & $-0.001(0.002)$ & $0.001(0.001)$ & $0.001(0.001)$ \\
\hline DL & $-0.296^{* *}(0.136)$ & $0.310(0.361)$ & $-0.346^{* *}(0.140)$ & $-0.259 *(0.143)$ \\
\hline Constant & $0.136^{* *}(0.054)$ & & & \\
\hline$W^{*}$ dep.var. & $0.269 *(0.139)$ & & & \\
\hline Spat.aut & & $-0.977 * * *(0.269)$ & $0.572 * * *(0.096)$ & $0.248(0.155)$ \\
\hline Teta & $\begin{array}{l}0.136 * * \\
(0.054)\end{array}$ & & & \\
\hline R-squared & 0.981 & 0.568 & 0.982 & 0.985 \\
\hline Likelihood & $-111,376.700$ & 133.721 & 532.622 & 544.512 \\
\hline LM-lag & $12.774 * * *$ & & & \\
\hline R-LM-lag & $76.380 * * *$ & & & \\
\hline LM-error & $3.230 *$ & & & \\
\hline R- LM-error & $66.836 * * *$ & & & \\
\hline Moran I & $0.038 * *$ & $0.038 * *$ & $0.038 * *$ & $0.038 * *$ \\
\hline Hausman-test & 2.938 & $-27.700 * * *$ & $-27.700^{* * *}$ & $-27.700 * * *$ \\
\hline
\end{tabular}

Note: $* * *, * *$ and $*$ represent significance at the $1 \%, 5 \%$ and $10 \%$ levels, respectively. The standard error is in brackets. Random: random effect model. Fixed: fixed effect model

regions. The eastern regions boast relatively developed economic conditions, high levels of marketization and relatively strong liquidity of factors. Therefore, given the comprehensive Internet infrastructure, Internet development can enhance the enterprise financing efficiency of eastern regions through the improvement of information asymmetry and the reduction of transaction costs. However, compared with central and western regions, central and western regions have the relatively backward economies, relatively low marketization and weak liquidity, which restricts the advantages of the Internet. Meanwhile, compared with eastern regions, the Internet infrastructure and supporting facilities in central and western regions shall be further perfected. Finally, the second assumption of this paper is further verified; the Internet has a spatial overflow effect on the improvement of enterprise financing efficiency. The regression results indicate that Internet development has a significant positive influence on enterprise financing efficiency at the national level. This result confirms the second assumption of this paper. With respect to analysis per region, the results of eastern regions indicate that Internet development also has a significant positive influence on enterprise financing efficiency. However, as for central and western regions, Internet development has no significant positive relationship with enterprise financing efficiency. This result is consistent with analysis of the 2SLS model. 


\subsection{Suggestions}

Since the eastern regions boast relatively developed economies and relatively comprehensive Internet infrastructure, it is not necessary to provide excessive financial inputs for the Internet infrastructure during the development of the Internet. However, eastern regions are under the dual influences of domestic and foreign economic environments due to their adjacency to the sea. Therefore, enterprises in eastern regions still face many financing restraints. Meanwhile, although eastern regions have relatively big financing demands and nongovernmental loan funds are abundant, enterprises' financing demands are often not matched with the supply of credit and loans due to the restrictions of financial policies. Thus, eastern regions require more policy dividends and the elimination of excessive unnecessary restrictions in the financial market. More preferential policies shall be formulated according to enterprises' actual demands. As a result, enterprises in eastern regions are able to acquire more financing information and channels through the Internet in order to overcome difficult enterprise financing. Compared with eastern regions, central and western regions are relatively underdeveloped, and the construction of the Internet infrastructure is incomplete. Moreover, the local economy significantly depends on financial support, and the overall scale of enterprise is not as big as that of eastern regions. Internet development does not have the foundation to thrive. Therefore, increasing financial inputs is now the top priority for the improvement of the construction of Internet infrastructure in central and western regions. Certainly, the technological opportunity is important for any region. On this basis, it is concluded in this paper that efforts including enhanced financial inputs and policy supports can be implemented to cultivate a batch of key pilot cities with strong economic influence in under-developed central and western regions for development of Internet. After all, it is unlikely to improve the development of the Internet in such regions in the short term. The application of some pilot financial policies in these regions can relieve the financing difficulties of enterprises in central and western regions but also avoid the occurrence of systematic financial risks in the economic growth stage of central and western regions.

Open Access This article is licensed under a Creative Commons Attribution 4.0 International License, which permits use, sharing, adaptation, distribution and reproduction in any medium or format, as long as you give appropriate credit to the original author(s) and the source, provide a link to the Creative Commons licence, and indicate if changes were made. The images or other third party material in this article are included in the article's Creative Commons licence, unless indicated otherwise in a credit line to the material. If material is not included in the article's Creative Commons licence and your intended use is not permitted by statutory regulation or exceeds the permitted use, you will need to obtain permission directly from the copyright holder. To view a copy of this licence, visit http://creativecommons.org/licenses/by/4.0/.

\section{References}

Akerlof GA (1970) The market for 'lemon': qualitative uncertainty and the market mechnism. Q J Econ 84: $488-500$

Bailey JP, Mcknight L (1997) Internet economics: what happens when constituencies collide?. Internet Economics. MIT Press, Cambridge

Charnes A, Cooper WW, Rhodes E (1978) Measuring the efficiency of decision making units. Eur J Oper Res 2(6):429-444 
Cheng L (2013) A study on innovation mechanism of Enterprise value network from the perspective of the internet economy. China Ind Econ 9:82-94

Cheng B, Ioannou I, Serafeim G (2014) Corporate social responsibility and access to finance. Strateg Manag J 35(1):1-23

Dobrovolsky SP (1958) Economics of corporate internal and external financing. J Financ 13(1):35-47

Du Zheng Siqi Y, Weizeng S (2016) Analysis of determinants and spatial variation of internet development in Chinese cities. Urban Dev Stud 23(12):11-14

Elhorst JP (2014) Spatial econometrics: from cross-sectional data to spatial panels. Springer, Berlin Heidelberg

Erel I, Jang Y, Weisbach MS (2015) Do acquisitions relieve target firms' financial constraints? J Financ 70(1):289-328

Fang L, Wang C (2014) System building of financing efficiency on innovative small and medium-sized enterprises. Stat Decision 2:172-175

Gang F, Wang X, Zhu H (2011) NERI INDEX. Economic Science Press, Beijing

Grange LD, Ibeas A, González F (2012) A hierarchical gravity model with spatial correlation: mathematical formulation and parameter estimation. Netw Spatial Econ 11(3):439-463

Griffith DA, Chun Y (2015) Spatial autocorrelation in spatial interactions models: geographic scale and resolution implications for network resilience and vulnerability. Netw Spatial Econ 15(2):337-365

Guangning T, Wang D (2014) The internet economy analysis of the financial innovation. Macroecon Manag 02:58-59

Haijian L, Yuexin T, Wenjie L (2014) Mobile internet thinking and traditional business reengineering. China Ind Econ 10:135-146

Kaplan SN, Zingales L (1997) Do investment-cash flow sensitivities provide useful measures of financing constraints? Q J Econ 112(1):169-215

Koutroumpis P (2009) The economic impact of broadband on growth: a simultaneous approach. Telecommun Policy 33(9):471-485

Lennox C, Wang ZT, Wu X (2017) Earnings management, audit adjustments, and the financing of corporate acquisitions: evidence from China. J Account Econ

Lichang L, Genfu F, Daohong Z, Mao H (2004) Efficiency evaluation on equity finance of listed companies on basis of DEA. Syst Eng 1:55-59

Manfeng L, Long Z (2019) Solving the Financing Constraints on Small and Micro Enterprises from the Perspective of Internet Finance. Manag Rev 31(3):39-49

Modigliani F, Miller MH (1958) The cost of capital, corporation finance and the theory of investment. Am Econ Rev 48(3):261-297

Modigliani F, Miller MH (1963) Corporate income taxes and the cost of capital: a correction. Am Econ Rev 53(3):433-443

Myers SC, Majluf NS (1983) Corporate financing decisions when firms have information investors do not have[J]. J Financ Econ 13(2):187-221

Pradhan RP, Arvin MB, Norman NR et al (2016) Financial depth, internet penetration rates and economic growth: country-panel evidence. Appl Econ 48(4):1-13

Quan Z (2014) A comparative study of financing efficiency on listed enterprises of small and medium-sized. Financial Theory \& Practice 4:42-45

Rajan RG (1992) Insiders and outsiders: the choice between informed and arm's-length debt. J Financ 47(4): 1367-1400

Samuelson PA (1952) The Transfer Problem and Transport Costs:The Terms of Trade when Impediments are Absent. Economic Journal, (62)278-304

Shouguo Z, Kong J, Sijia L (2011) Analysis of financing efficiency of listed companies in Shaanxi Provincebased on DEA Models. China Soft Sci s2(2011):245-253

Stiglitz JE, Weiss A (1981) Credit rationing in markets with imperfect information. Am Econ Rev 71(71):393-410

Tone K (2001) A slacks-based measure of efficiency in data envelopment analysis. Eur J Oper Res 143:32-41

Wang X (2009) Information asymmetry, Enterprise characteristics and credit rationing in commercial banks. J Zhongnan Univ Econ Law 3:73-77

Wang X, Huiping D, Hu yi. (2017) The evaluation of financing efficiency for China's small and medium-sized enterprises based on DEA model. Syst Eng Theory Practice 37(4):865-874

Wooldridge JM (2014) Introductory econometrics: a modern approach. Tsinghua University Press, Beijing

Xinmin Z, Tingting Z, Deqiu C (2017) Industrial policy, financing constraints and investment efficiency. Accounting Research. (4):12-18 
Han Xuwen. (2016). Assessment of Enterprise financing efficiency based on DEA model - taking listed companies of NEEQ for example. Shandong University

Yichao L (2003) The empirical analysis of financing structure of listed companies in China. Quant Tech Econ 20(6):147-150

Yu L, Yu Q, Gao Q (2013) Evaluation on real estate Companies's debt financing efficiency based on the threestage DEA model. Sci Res Manag 34(8):147-157

Yuan Q, Zhao Y, Shang H, Zhang W, Umar Z (2016) Financing constraints on the size distribution of industrial firms: the Chinese experience. Appl Econ 41

Zhiqiang L (2015) Study on relationship between internet finance and SMEs' financing from the perspective of transactions cost. Shanghai Econ Rev 3:67-73

Publisher's note Springer Nature remains neutral with regard to jurisdictional claims in published maps and institutional affiliations. 